Review

\title{
lncRNAs and microRNAs with a role in cancer development
}

\author{
Julia Liz ${ }^{\text {a }}$, Manel Esteller ${ }^{\mathrm{a}, \mathrm{b}, \mathrm{c}, *}$ \\ ${ }^{a}$ Cancer Epigenetics and Biology Program (PEBC), Bellvitge Biomedical Research Institute (IDIBELL), L'Hospitalet, Barcelona, Catalonia, Spain \\ b Department of Physiological Sciences II, School of Medicine, University of Barcelona, Barcelona, Catalonia, Spain \\ c Institucio Catalana de Recerca i Estudis Avançats (ICREA), Barcelona, Catalonia, Spain
}

\section{A R T I C L E I N F O}

\section{Article history:}

Received 1 April 2015

Received in revised form 3 June 2015

Accepted 30 June 2015

Available online xxxx

\section{Keywords:}

RNA

Non-coding RNAs

Transcribed ultraconserved regions MicroRNA

Cancer

\begin{abstract}
A B S T R A C T
Most diseases, including human cancer, are frequently associated with an altered transcription pattern. The alteration of the transcriptome is not restricted to the production of aberrant levels of protein-coding RNAs, but also refers to the dysregulation of the expression of the multiple noncoding members that comprise the human genome. Unexpectedly, recent RNA-seq data of the human transcriptome have revealed that less than $2 \%$ of the genome encodes protein-coding transcripts, even though the vast majority of the genome is actively transcribed into non-coding RNAs (ncRNAs) under different conditions. In this review, we present an updated version of the mechanistic aspects of some long non-coding RNAs (IncRNAs) that play critical roles in human cancer. Most importantly, we focus on the interplay between IncRNAs and microRNAs, and the importance of such interactions during the tumorigenic process, providing new insight into the regulatory mechanisms underlying several ncRNA classes of importance in cancer, particularly transcribed ultraconserved regions (T-UCRs).
\end{abstract}

(c) 2015 Elsevier B.V. All rights reserved.

\section{The non-coding RNA landscape}

Almost the entire mammalian genome is actively transcribed under specific conditions [1]. Many of the resulting transcripts are processed to give rise ultimately to small RNAs. The range of types of small RNA includes microRNAs, which are the most extensively studied class of non-coding RNAs (ncRNAs). MicroRNAs are the best-known subtype and it is widely accepted that their function is crucial during developmental processes, apoptosis and cell proliferation [2]. MicroRNAs posttranscriptionally regulate the expression of their target genes through their complementarity with the mRNA sequence. The generation of functional microRNAs is a process that transforms the long primary transcript into a mature functional $\sim 22 \mathrm{nt}$ microRNA. MicroRNA genes are embedded within intronic genomic regions or within the exons of protein-coding genes [3]. They are initially transcribed by RNA polymerase II as long, capped and polyadenylated primary transcripts (pri-microRNAs) that may contain more than one hairpin structure, known as polycistronic microRNA clusters [4]. In the canonical processing pathway, pri-microRNAs are recognized and cleaved by the Microprocessor complex, which contains the RNase III enzyme Drosha and DGCR8 as a cofactor $[5,6]$. The cleaved portion of the hairpin (premicroRNA) needs to be exported to the cytoplasm to complete its processing by the cytoplasmic RNAse III enzyme Dicer, leaving the $\sim 22 \mathrm{nt}$ duplex [7]. The guide strand is then joined to one of the Ago proteins to establish the core of the silencing complex, whereas the passenger

* Corresponding author at: Cancer Epigenetics and Biology Program (PEBC), Bellvitge Biomedical Research Institute (IDIBELL), L'Hospitalet, Barcelona, Catalonia, Spain.

E-mail address: mesteller@idibell.cat (M. Esteller). strand is discarded $[8,9]$. The mature RISC complex can bind the untranslated region (UTR), generally the 3'-UTR, but some recent reports have shown that microRNA targeting is not always limited to $3^{\prime}$-UTR, since, with respect to the $5^{\prime}$-UTR of mRNAs, there are some naturally occurring examples in which the targeting microRNA downregulates the expression of the corresponding mRNA in a seed-dependent manner. This highlights the complexity of microRNA targeting in the posttranscriptional control of oncogenes or tumor suppressor genes [10]. Finally, microRNA-mRNA binding leads to translational inhibition or degradation of the target mRNA [11].

Considering that most mammalian protein-coding genes are regulated by at least one microRNA, and that a vast number of nonconserved sites exists, it is not unexpected that their function and biogenesis are firmly regulated, since their alteration is associated with a wide range of human diseases, including cancer and neurological disorders [12]. In this regard, the final amount of each microRNA can be regulated at the transcriptional or post-transcriptional level. As with protein-coding genes, most microRNAs are transcribed by RNA Pol II and can be regulated at the transcriptional level since they feature canonical promoters and enhancers in their regulatory upstream regions [13]. At this level, it is well established that their expression depends on the activity of transcription factors such as c-Myc and p53 $[14,15]$, or on epigenetic modifications, such as DNA methylation and histone modifications, that occur at their promoter sequences [16]. Since microRNA biogenesis is a multistep process, any step could be tightly regulated. Moreover, in recent years, many models have revealed some post-transcriptional mechanisms that could account for the discrepancies between the expression levels of pri-microRNAs and the corresponding mature microRNAs [17]. Among the post- 
transcriptional mechanisms that regulate the final expression of any microRNA, the control of the Microprocessor complex stands out since the effectiveness of the catalytic process mediated by Drosha is crucial for determining microRNA abundance. Many mechanisms exist and these have been summarized in some excellent reviews [18,19]. However, to date, these have only been concerned with protein factors bound to distinct elements along the pri-microRNA or that interact with the Drosha-DGCR8 to control microRNA maturation [20,21]. Very recently, our group has identified a new mechanism for the posttranscriptional control of microRNAs at the level of Drosha processing. It is mediated by a long non-coding RNA (lncRNA) transcribed from an ultraconserved region, which highlights the great complexity of ncRNA regulatory networks between different ncRNA classes of importance in cancer [22]. This example and others are considered in detail in the IncRNA:microRNA section. Overall, it has been shown that many microRNAs are regulated during the catalytic process mediated by Drosha, and that this regulation represents a dominant mechanism that has consequences for microRNA expression during development and in cancer [23].

The IncRNA category comprises a very heterogeneous subclass that was first described by genome-wide sequencing of cDNA libraries of the mouse genome. It is becoming clear that a large number of IncRNAs are determining regulators of normal tissue physiology and the processes of diseases such as cancer and neurological disorders, despite so often being branded as transcriptional noise. As a result, much effort is currently being made to characterize those genetic, epigenetic and transcriptional changes that occur during disease. The methods used increasingly involve high-throughput RNA-seq technologies designed to enhance our knowledge of cancer pathogenesis [24,25]. In this context, very recent findings have revealed an expanding landscape of transcription via unbiased transcriptome reconstruction from thousands of tumors, normal tissues and cell lines, showing that the number of IncRNAs has been hitherto underestimated. This new assembly clearly demonstrates that the genomic heterogeneity of lncRNAs eclipses that of coding transcripts $(60,000$ lncRNA genes versus roughly 30,000 protein-coding genes), a discrepancy that may grow since additional diseases and cell types are currently being sequenced and more lncRNAs are being identified. Although the functions of the vast majority of IncRNAs have not been validated, it is thought that many of these transcripts could serve as future cancer biomarkers [26].

\section{Long non-coding RNAs at the heart of gene regulation in health and disease}

Every eukaryotic cell relies on a highly integrated gene expression program whose integrity is ensured by transcriptional and posttranscriptional processes. Some years ago, IncRNAs were first recognized as being crucial regulators of gene expression in a wide range of biological contexts, even though the mechanisms by which the vast majority exert their functions remain largely unknown. IncRNAs are a very heterogeneous group of transcripts longer than $200 \mathrm{nt}$ that have no protein-coding capacity. Unlike microRNAs, the length of lncRNAs allows them to fold into intricate structures, by which they may perform their function as RNA sequences by themselves through secondary and tertiary structural determinants. In general, IncRNA transcripts exhibit low-level but tissue-specific expression [27] and, with some exceptions [22], their nucleotide sequence is poorly conserved [28]. In the last few years, several studies have shown the importance of lncRNAs to normal physiology as well as to the control of gene expression, wherein they modulate key cellular processes such as cell proliferation, senescence, migration and apoptosis [29,30].

The intrinsic ability of IncRNAs to interact with DNA, RNA and proteins by acting as guides, tethers, decoys and scaffolds offers the most compelling explanation of their ability to regulate gene expression, including epigenetic transcriptional control by their association with chromatin remodeling complexes [31], splicing [32,33], translation
[34] and protein stability. Moreover, an increasing body of experimental evidence suggests connections between microRNAs and lncRNA. A new function for lncRNAs has become apparent from their ability to regulate microRNA activity by acting as either competitive endogenous RNAs for microRNAs or as microRNA sponges [35-37].

Considering that lncRNAs control key cellular processes, knowledge of the mechanisms by which lncRNA expression is regulated is the first step towards understanding the basic principles by which they exert their functions. Furthermore, many studies have shown that lncRNA expression is altered in a variety of human cancer types [38] and that their expression pattern may be associated with metastasis and disease prognosis $[39,40]$. The expression of specific lncRNAs with oncogenic features $[41,42]$ is closely linked to the ability to promote matrix invasion of cancer cells and tumor growth [43].

In spite of their importance, the mechanisms that govern the control of IncRNAs are largely unknown but there is some evidence that demonstrate that they are regulated by a similar mechanism to that controlling protein-coding genes and microRNAs. However, there are some discrepancies about the transcriptional regulation of lncRNAs by epigenetic mechanisms, especially DNA methylation. In this context, genome studies involving 5Cm, H3K4me3, H3K9me3, H3K27me3 and H3K36me3 epigenetic marks associated with transcription start sites of IncRNAs common to various human cell types showed that the pattern of DNA methylation and H3K9me3 differs considerably between mRNAs and lncRNAs and that it does not seem to play a role in IncRNA expression [44].

Despite this disparity, it has been clearly demonstrated that, in addition to microRNAs, another singular class of IncRNAs transcribed from ultraconserved regions of the genome is also subject to transcriptional silencing driven by epigenetic mechanisms such as DNA methylation and histone modifications. This highlights the importance of epigenetic disruption of IncRNA in human tumorigenesis [45].

As with proteins, over the past decade many studies have shown that some lncRNAs are under the regulation of microRNAs to reduce their stability (Table 1). Given that the proper activity of these lncRNAs is essential to key cellular processes, changes in their expression can alter diverse molecular pathways altering physiological processes. In this context, the role of HOTTIP has been found to be the most significant oncogenic lncRNA in human hepatocellular carcinoma (HCC). Moreover, miR-125b binds to one putative microRNA binding site in the HOTTIP sequence by acting as post-transcriptional regulator of HOTTIP expression [46]. Another well characterized lncRNA whose activity is affected by microRNA function is linc-p21 RNA, which is transcriptionally activated by p53. MiR-let7b, in cooperation with the RNA

Table 1

Examples of cancer associated lncRNAs and T-UCRs post-transcriptionally regulated by microRNAs.

\begin{tabular}{|c|c|c|c|}
\hline & microRNA & Disease/process & Ref \\
\hline \multicolumn{4}{|l|}{ LncRNA } \\
\hline HOTTIP & miR-125b & Hepatocellular carcinoma & Tsang et al. [46] \\
\hline Linc-p21 & Let-7b & Cervical carcinoma & Yoon et al. [47] \\
\hline \multirow[t]{2}{*}{ MALAT1 } & $\operatorname{miR}-9$ & $\begin{array}{l}\text { Glioblastoma and Hodgkin's } \\
\text { lymphoma }\end{array}$ & Leucci et al. [48] \\
\hline & miR-101 & $\begin{array}{l}\text { Esophageal squamous } \\
\text { carcinoma }\end{array}$ & Wang et al. [49] \\
\hline loc285194 & miR-211 & Colorectal cancer & Liu et al. [19] \\
\hline CASC2 & miR-21 & Endometrial cancer and gliomas & Baldinu et al. [51] \\
\hline UCA1 & miR-1 & Bladder carcinoma & Fan et al. [55] \\
\hline HOTAIR & miR-141 & Renal carcinoma & $\begin{array}{l}\text { Chiyomaru et al. } \\
{[42]}\end{array}$ \\
\hline \multicolumn{4}{|l|}{$T$-UCR } \\
\hline Uc. $160+$ & $\begin{array}{l}\operatorname{miR}-155 \\
\text { miR-24-1 }\end{array}$ & $\begin{array}{l}\text { Chronic lymphocytic leukemia } \\
\text { and carcinomas }\end{array}$ & Calin et al. [84] \\
\hline Uc. $346+A$ & miR-155 & & \\
\hline Uc.78+ & $\begin{array}{l}\text { miR-15a } \\
\text { miR-16 }\end{array}$ & & \\
\hline
\end{tabular}


binding protein HuR contributes to reduce linc-p21 activity in human cervical carcinoma cell lines [47].

The abundant, ubiquitously expressed and highly conserved lncRNA MALAT-1 (Metastasis-Associated Lung Adenocarcinoma Transcript 1) is also targeted by miR-9 in an Ago2 complex-dependent manner in the nucleus of glioblastoma and Hodgkin cell lines. The regulation of these two ncRNA species relies on two binding sites located along MALAT-1 sequence, emphasizing the complexity of microRNA activity that is not restricted solely to the cytoplasm [48]. In addition, in spite of being deregulated in many types of cancer, its role in esophageal squamous carcinoma (ESCC) has been demonstrated very recently. Wang and coworkers found that the post-transcriptional silencing of MALAT1 by miR-101 and miR-217 leads to a significant reduction in proliferation by the arrest of the $\mathrm{G} 2 / \mathrm{M}$ cell cycle that is explained by the deregulation of downstream effectors of MALAT1, such as MIA2, HNF4G, ROBO1, CCT4 and CTHRC1 [49].

The lncRNA loc285194 and its relationship with miR-211 gives rise to a repression feedback loop. As we describe below, loc285194 harbors two binding sites for miR-211 that can function as an endogenous sponge for miR-211. Additionally, loc285194 activity is also affected by the ectopic expression of miR-211 [50]. In this regard, it has been suggested that the lack of function of p53-inducible loc285194 in colon tumors is triggered by the upregulation of miR-211 when its expression is enhanced.

Tumor-suppressor properties for the IncRNA CASC2 (cancer susceptibility candidate 2 ) have recently been identified in endometrial cancer [51] and in human gliomas. In the case of gliomas, the oncogenic miR-21 binds to the CASC2 IncRNA sequence in an Ago2-dependent manner, reducing its expression and thereby the CASC2-mediated inhibition of glioma cell proliferation, migration and invasion. It has also been suggested that reciprocal regulation is also possible, by which the ectopic expression of CASC2 suppresses the malignancy of glioma cells mainly by miR-21. CASC2 IncRNA has been postulated as being one of the IncRNAs that act as endogenous microRNA sponges, as described below [44].

UCA1 IncRNA (urothelial carcinoma-associated 1) is significantly upregulated in most tumors and cancer cells, including colorectal cancer [52], tongue squamous cell carcinomas [53], breast cancer [54] and bladder cancer [55]. In bladder carcinomas, in which miR-1 is downregulated, UCA1 lncRNA is known to harbor predictable binding sites for this microRNA. In doing so, miR-1 reduces the expression of UCA1 IncRNA in an Ago2-slicer-dependent manner. The relation between the two types of non-coding RNAs has been demonstrated with luciferase-based reporter assays [56].

\section{Mechanisms of gene regulation by long non-coding RNAs}

By definition, the transcriptional regulation mediated by lncRNAs is the consequence of the assembly of the transcription machinery resulting from the action of transcriptional factors on enhancer and promoter regions. In this context, IncRNAs may be involved in the transcriptional regulation either as cis- or trans-acting elements, and could negatively or positively influence gene expression.

\section{Cis-acting long non-coding RNAs}

Transcriptional regulation by cis-acting elements implies when the regulatory lncRNA and the target gene are both transcribed from the same locus [57]. However, some recent findings suggest that cis-acting IncRNA also has the ability to act in trans [58-60], highlighting the importance of DNA loop formation in mediating the regulation of the two RNAs. One of the best examples of this first category is the human HOTTIP IncRNA, which is transcribed from the HOXA cluster and induces transcription of neighboring protein-coding genes by its interaction with WDR5 and the MLL histone modifier complex, which adds histone $\mathrm{H} 3$ lysine-4 trimethylation (H3K4me3) over the flanking gene promoters [61]. Correlations between clinico-pathological parameters and levels of expression in hepatocellular carcinoma samples have demonstrated that the levels of HOTTIP transcript are associated with clinical progression of HCC patients and predict disease outcome, highlighting the role of this transcript in HCC and hence suggesting that HOTTIP is a predictive biomarker of this disease [62].

Another example of a cis-acting IncRNA is the non-spliced PANDA transcript, which is transcribed from a 5 -kb upstream promoter region of the CDKN1A cell-cycle gene, and is activated in response to DNA damage [63]. PANDA IncRNA, by interacting with polycomb repressive complexes and the transcription factor NF-YA, endows cells with the capacity to maintain their proliferative state, and alterations in their levels can lead to entry or exit from the senescent state [64].

Global transcriptome analysis has revealed that 70\% transcripts have antisense counterparts, known as natural antisense transcripts (NATs), whose transcription can activate or inactivate the sense gene [65]. Consistent with their genomic locations, members of this group can be subclassified into cis-NATs and trans-NATs. In the first category, ANRIL IncRNA stands out because it is transcribed from the same gene-cluster locus that codes for the tumor suppressors $C D K N 2 A$ and CDKN2B [66]. ANRIL transcripts are associated with CBX7, which has the ability to recognize the repressive histone mark H3K27me3 and to keep the target gene cluster silenced. With respect to functionality, it has very recently been reported that lncRNA ANRIL plays an important role in non-small cell lung cancer outcomes since its expression is closely associated with tumor-node metastasis stage and tumor size. The loss of expression of ANRIL induces apoptosis in vivo and in vitro, providing novel insights into the functionality of this IncRNA transcript in tumorigenesis [67].

\section{Trans-acting elements}

Increasing numbers of lncRNAs are known to be direct interactors with specific regions of the chromatin located across multiple different chromosomes to modulate gene expression at the genomic level. It is well known that trans-acting lncRNAs target proteins, distal chromatin regions of even others ncRNAs while the determining factor for these long-range interactions is not well established. Almost all classes of RNA molecules are governed by a characteristic secondary structure. Since this RNA architecture allows RNA to recruit and interact with proteins, other nucleic acids as well as with itself, deciphering the RNA structural language and understanding the structure-function relationship is critical for a deeper knowledge of trans-acting RNA function, especially for lncRNAs for which no common function is known. Some studies have suggested that the intrinsic architecture of lncRNAs is the key factor determining specific interactions $[68,69]$.

In this category, two lncRNAs that are highly overexpressed in aggressive prostate cancer, PRNCR1 (also known as PCAT8) and PCGEM1, activate the transcription of ligand-dependent or ligandindependent androgen receptors by binding to androgen receptors, thereby enhancing proliferation programs in prostate cancer cells as was reported by Rosenfeld group in 2013. Binding of PRNCR1 to the acetylated androgen receptor is required for the sequential association with the H3K79 methyltransferase DOT1L that in turn, and is necessary for the recruitment of the second IncRNA involved in the present mechanism, PCGEM, which is methylated by DOT1L [70].

\section{Competing endogenous RNA network: microRNA sponges and decoys}

As mentioned above, IncRNAs may exert their function through transcriptional or post-transcriptional mechanisms. Some years ago, a new regulatory network, first described in plants [71], has emerged in the post-transcriptional control of gene expression by characterizing the ability of numerous IncRNA transcripts to compete for microRNA binding, thereby alleviating the negative effect of microRNAs on 
their respective mRNA targets. These RNA transcripts are commonly known as competing endogenous RNAs (ceRNAs) or natural microRNA sponges. This category includes IncRNAs, circular RNAs and pseudogenes. The discovery of the ceRNA network reveals an additional layer of post-transcriptional regulation and, most importantly, reveals a complexity of ncRNA regulation of various non-coding RNA species of importance in cancer. As we mentioned before, almost all classes of RNA molecules are governed by an intrinsic secondary architecture that allows RNA to recruit and interact with proteins, other nucleic acids as well as with itself. Based on the principle of complementarity, Steitz and coworkers proposed the hypothesis, based on computational methods, that the existence of microRNA binding sites could be titrating specific microRNA and thus, affecting microRNA availability and, in turn, affecting to downstream processes [72]. Poliseno and collaborators subsequently demonstrated experimentally that the pseudogene derived from the PTEN locus sequesters microRNA due to their highly similar sequence. They showed that the expression of the ancestral proteincoding transcript was subordinated to the expression of the pseudogene transcript (PTENP1) by acting as a sponge for miR-19b and miR-20a binding sites shared between these two transcripts [73]. These bona fide microRNA competitors actively compete with their ancestral protein-coding genes for the same pool of microRNAs and so are able to regulate their relative abundances. This highlights how various non-coding RNA elements in a network may communicate with each other through microRNA Response Elements (MREs). Apart from the PTENP1 RNA transcript, many other transcripts, most of which are messenger RNAs, are known to be competing endogenous RNAs. Our review is concerned only with those lncRNAs that have the capacity to alter microRNA activity through the presence of MREs in their sequences. A handful of IncRNAs have so far been identified as decoys for microRNAs; some of these are illustrated in Table 2.

The H19 IncRNA is a highly conserved and imprinted transcript, $2.6 \mathrm{~kb}$ long, that is mainly found in the cytoplasm and is known to have an important role in growth [74]. This oncogenic lncRNA transcript harbors canonical and non-canonical binding sites for some members of the let-7 family, regulating their expression, which has crucial roles in cancer [75]. The functionality of this regulation was validated by genome-wide transcriptome analysis and by several in vivo experimental approaches to conclude that H19 knockdown releases let-7, leading to a stronger let-7 function [76].

Papillary thyroid carcinoma susceptibility candidate 3 (PTCSC3) is a recently discovered tumor suppressor lncRNA that is dramatically downregulated in thyroid cancers. After an in silico analysis, 20 putative binding sites for different microRNAs were identified along the PTCSC3 sequence. Of these, miR-574-5p was identified as a specific target for this lncRNA. The significant inverse correlation between PTCSC3 and miR-574-5p suggests that PTCSC3 acts as a molecular decoy to target the oncogenic miR-574-5p that consequently regulates cell growth

Table 2

Examples of cancer associated lncRNAs and T-UCRs that post-transcriptionally target microRNAs.

\begin{tabular}{llll}
\hline & microRNA & Disease/process & Ref \\
\hline LncRNA & & & \\
H19 & Let-7 & Pancreatic carcinoma & Ma et al. [75] \\
PTCSC3 & miR-574 & Thyroid cancer & Fan et al. [77] \\
CASC2 & miR-21 & Glioma & Wang et al. [44] \\
HOST2 & Let-7b & Ovarian cancer & Gao et al. [78] \\
LincRNA-RoR & miR-145 & Endometrial cancer stem cells & Zhou et al. [80] \\
& miR-205 & Breast cancer & Hou et al. [97] \\
HULC & miR-372 & Hepatocellular carcinoma & Wang et al. [81] \\
loc285194 & miR-211 & Osteosarcoma tumors & Liu et al. [50] \\
ncNRFR & Let-7 & Colonic malignant transformation & Franklin et al. [82] \\
& & & \\
T-UCR & & & Liz et al. [22] \\
Uc.283+A & miR-195 & Colorectal cancer and & \\
& & neuroblastoma &
\end{tabular}

and apoptosis in thyroid cancer. The discovery that PTCSC3 regulation is part of miR-574's activity sheds some light on the molecular mechanisms that underlie thyroid cancer and provides new molecular markers for its earlier diagnosis [77].

As explained above, miR-21 post-transcriptionally regulates CASC2 function in a sequence-specific manner, but a negative feedback loop mediated by the RNA-induced silencing complex (RISC) has also been reported [51]. In this regard, CASC2 expression is dramatically reduced in glioma tissues, promoting the migration and proliferation of malignant cells. In silico and in vivo experimental validations using techniques such as luciferase assays and RNA pull-downs confirmed that CASC2 IncRNA behaved as a microRNA sponge for miR-21 in U251 and U87 glioma cell lines that overexpressed this lncRNA through one highly conserved MRE. Therefore, CASC2 could be a new candidate therapeutic target gene for treating gliomas [44].

Intriguingly, the regulation between the evolutionarily conserved IncRNA HOST2 (human ovarian cancer-specific transcript 2) and the microRNA let-7b also works in both directions. The HOST IncRNA binds to microRNA let-7b, a potent tumor suppressor microRNA, thereby mediating post-transcriptional regulation. HOST2 RNAs have only one binding site for let-7b, recruiting it and modulating its accessibility to its natural targets by acting as a molecular decoy. HOST2 can affect let-7b functions, which help to enhance the oncogenic features bestowed on HOST2 IncRNA by accentuating the endogenous expression of metastasis-promoting genes such as HMGA2, c-Myc and Imp3 [78].

LincRNA-RoR has been reported to act as a molecular decoy for miR145 , which in turn regulates the mRNA levels of core transcription factors such as Oct4, Sox2, and Nanog, to maintain cell renewal in human stem cells (hESCs) [79]. In doing so, the linc-RoR regulates miR-145 expression in a sequence-dependent manner, since only the overexpression of the wild-type form of the IncRNA had any effect on microRNA function. Moreover, the overexpression of linc-RoR affects the mature levels of miR-145, whereas the precursor levels were unaffected, confirming the action of the post-transcriptional mechanism [79]. Subsequently, the negative regulation of miR-145 by linc-RoR in endometrial cancer stem cells was demonstrated, which suggests that linc-RoR functions could be similar to the mechanism described in hESCs. This highlights the importance of this regulatory feedback loop in physiological aspects of early carcinogenesis [80].

In a similar manner, linc-ROR overexpression leads to an epithelialto-mesenchymal transition (EMT) phenotype in mammary cells, emphasizing its role in tumorigenesis. In this study, Hou and coworkers showed that, in addition to miR-145, linc-RoR also functions as a microRNA sponge for miR-205. In doing so, linc-RoR prevents the downregulation of miR-205 target genes such as ZEB2, which is known to cause EMT and metastasis in breast cancer.

One of the most strongly upregulated genes in hepatocellular carcinoma is the lncRNA HULC, whose depletion causes some alterations in genes involved in liver cancer. In this particular case, HULC RNA has been described as a competing endogenous RNA for miR-372 that mediates the post-transcriptional repression of PRKACB genes which finally phosphorylates CREB, which in turn upregulates HULC [81].

Another good example of microRNA titration is the mutual relation between loc285194 RNA and miR-211 that involves the RISC complex. loc285194 is a p53-regulated tumor suppressor lncRNA that harbors two binding sites for miR-211 that explains its tumor suppressor features in osteosarcoma tumors [50].

The characterization of the ncNRFR IncRNA in colonic homeostasis by Franklin and coworkers has elucidated the functional role of lncRNA species. This lncRNA is transcribed from the RAS locus but appears to be an independent transcriptional unit. Their study found that the overexpression of this ncNRFR transcript in non-malignant cells gives rise to a highly invasive and metastatic phenotype. It also reported a functional interaction between ncNRFR transcript and let-7 family, in which the complementary sequences are outside of the seed region. Bearing in 
mind the importance of the role of let-7 family members as tumor suppressors, any alteration would have important associations with a malignant phenotype. Using in vitro validation based on luciferase reporter assays upon ncNRFR overexpression in non-malignant cells, these authors showed that ncNRFR-positive cells ameliorate the repression of the let-7 reporter relative to controls, indicating that the ncNRFR transcript downregulates let-7 activity. Further analysis of let-7 targets has demonstrated that they are significantly upregulated by ncNRFR expression. Thus, the ncNRFR transcript inhibits let-7 function, disrupting the growth of normal colonic epithelial cells [82].

Last, but not least, an important mechanism in the posttranscriptional control of microRNAs by lncRNAs has been described very recently by our group. In this particular case, an IncRNA transcribed from an ultraconserved region of the genome impairs Microprocessor recognition by binding to the primary microRNA-195 transcript through sequence complementarity. A detailed explanation of the mechanism underlying this first reported case of RNA-directed regulation of microRNA biogenesis is provided in the next section [22].

\section{IncRNAs harboring conserved elements in cancer: T-UCRs}

Nowadays, the degree of conservation of IncRNAs has been a controversial topic. In this respect, an intriguing feature of the non-coding genome includes ultraconserved regions (UCRs), otherwise known as ultraconserved elements (UCEs). These genomic regions describe a subset of 481 conserved sequences longer than 200 bp that are fully conserved between orthologous regions of the mouse, rat and human genomes [83]. Due to their degree of conservation, it is possible that these 481 UCRs contain functional elements [83] that are essential to the physiology of the cell, although to date, their biological roles remain largely uncharacterized. Approximately $25 \%$ of them overlap with the mRNA of protein-coding genes and are known to be exonic elements. By contrast, the non-exonic elements are usually clustered within gene deserts and it has been suggested that they may be involved in the regulation of transcription at the DNA level, acting as enhancers of distal neighboring protein-coding genes [83].

A high percentage of these UCRs ( $93 \%)$ are actively transcribed as RNAs products, and are known as T-UCRs, exhibiting ubiquitous and tissue-specific expression patterns that are very often altered in human carcinomas [84]. Analyzing their expression profiles makes it possible to distinguish human cancers. Among these aberrantly expressed T-UCRs, Uc.338+ is of particular note with respect to the pathobiology of hepatocellular carcinoma (HCC), since it is dramatically upregulated compared with adjacent non-cancerous tissues. A decrease in the anchorage-dependent and anchorage-independent growth of HCC cells was observed upon Uc.338+ knockdown, highlighting the role of this T-UCR in regulating cell transformation and suggesting that Uc.338+ is a genetic marker of susceptibility in HCC [85].

T-UCR expression also seems to be important in prostate cancer, such that a significant number of T-UCRs were differentially expressed between tumor and non-cancerous tissues. In this context, Uc.363+A and Uc.454+A appear to be the most significantly upregulated and downregulated T-UCRs, respectively. Uc.160+ also plays an important role since its downregulation has generated a robust gene expression profile in prostate LNCaP cells in which numerous genes that cluster in proliferation and cell movement pathways are upregulated or downregulated relative to control cells, suggesting that Uc.106+ is a functional transcript that may have an important role in prostate cancer [86].

It is well known that colorectal cancer (CRC) develops as a result of multiple genetic alterations. In the study carried out by Sana et al., Uc.73+ and Uc.388 + were identified as potential players in the diagnosis and prognosis of CRC patients, since they exhibit lower levels of expression than non-tumoral tissue samples, suggesting that both had tumor-suppressor properties [87]. However, the functional role of Uc.73+ in CRC is a controversial topic that is yet to be resolved. Uc.73+ has been described as one of the most frequently upregulated
T-UCRs in colon cancer and the effects of this downregulation have been studied by Calin and coworkers. Upon Uc.73+ downregulation, a significant decrease in COLO-320 cell growth and an increase in the sub-G1 fraction was observed, which suggests the presence of apoptosis in these cells in which this T-UCR is upregulated.

UCRs are often located at fragile sites and genomic regions affected in various cancers (cancer-associated genomic regions, CAGRs). This is the case, for instance, for Uc.349+A and Uc.352+, whose expression differs between normal and leukemic CD5-positive cells [84]. Both TUCRs are immersed within the 13q21.33-q22.2 genomic region that has been associated with susceptibility to familial chronic lymphocytic leukemia [88].

As mentioned above, the alteration of T-UCR expression in cancer may be achieved by hypermethylation of $\mathrm{CpG}$ islands in their respective promoter regions [45] (Fig. 1) or by their interaction with microRNAs [84]. Concerning the epigenetic mechanisms that control T-UCR expression at the transcriptional level, the presence of $\mathrm{CpG}$ island promoter hypermethylation is associated with T-UCR silencing in a variety of tumor types and human cancer cell lines. As briefly described above, Uc.283+A, Uc.346+ and Uc.160+ are subject to epigenetic silencing associated with the hypermethylation of promoters. In such a way, after treatment with the DNA demethylating agent 5-Aza-2'deoxycytidine (5-AzaC), these T-UCRs lose methylation in their promoter regions and undergo a concomitant restoration of their expression levels. Furthermore, Uc.160+, Uc.283+A and Uc.346+ hypomethylation was linked to a more accessible chromatin conformation of the RNA polymerase II enzyme and was accompanied by the presence of the active histone mark H3K4me3 [45].

Hudson and coworkers also found six T-UCRs underwent transcriptional silencing driven by epigenetic mechanisms such as DNA methylation. In particular, they found that Uc.308+A, Uc.434+A, Uc.241+A, Uc.283+A. Uc.285+ and Uc.85+ expression was recovered upon 5-AzaC and/or the histone deacetylase trichostatin A treatment in prostate cancer cell lines [86]. Of these, Uc.283+A had been previously found to be silenced by CpG hypermethylation in HCC cells [45].

Some instances of interactions between T-UCRs and microRNAs have been described and are discussed extensively in the following sections. Their intrinsic capacity to form RNA duplexes with microRNAs may represent a mechanism of mutual regulation that could work in both directions.

\section{T-UCR targets for microRNAs}

In 2007, Calin and collaborators demonstrated for the first time that some T-UCRs underwent altered expression patterns due to their direct regulation by highly expressed microRNAs in chronic lymphocytic leukemia (CLL). In particular, they confirmed that Uc.160+, Uc.346+A and Uc.348+ showed significant antisense complementarity with the miR-29a, miR-29b, miR-29c, miR-155, miR-146, miR-223 and miR-24 microRNAs, in which the expression of each T-UCR was negatively correlated with the corresponding microRNA levels. Each T-UCR:microRNA pair was extensively validated in vivo by measuring the expression levels of Uc.160+ and Uc.346 + A after the overexpression of miR-155 in leukemia cells in which both T-UCRs were downregulated. Furthermore, Uc.348 + was cloned into luciferase reporter vectors to verify its interaction with miR-155, miR-24-1 and miR-29-b in vitro, in which a concomitant reduction in luciferase expression was observed. This confirms the association between the two RNA species and, most importantly, suggests that an ncRNA network underlies the tumoral phenotype.

This fortuitous discovery and the high degree of conservation of TUCRs that hints at their biological function prompted researchers to investigate T-UCR functionality. They found altered patterns of T-UCR expression that are associated with specific tumor types. For instance, Scaruffi et al. showed that a negative correlation between T-UCRs and microRNAs activity may play an important role in the pathogenesis of 
NORMAL CELL

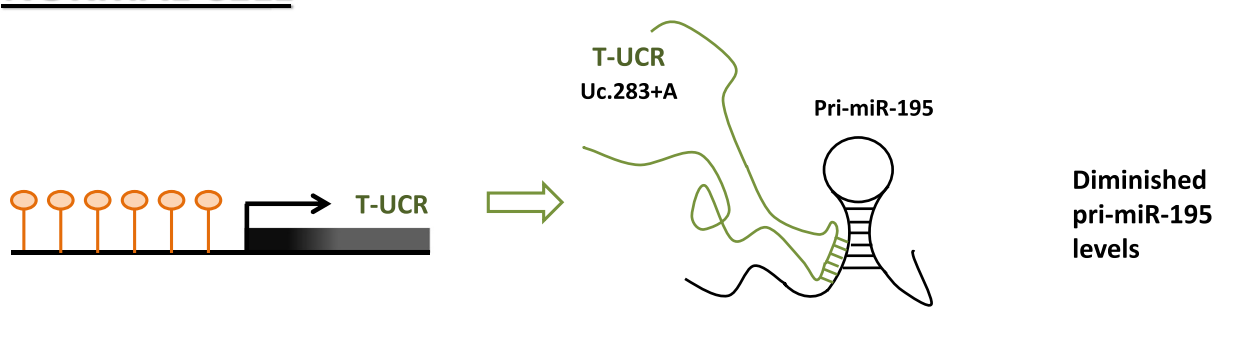

\section{CANCER CELL}

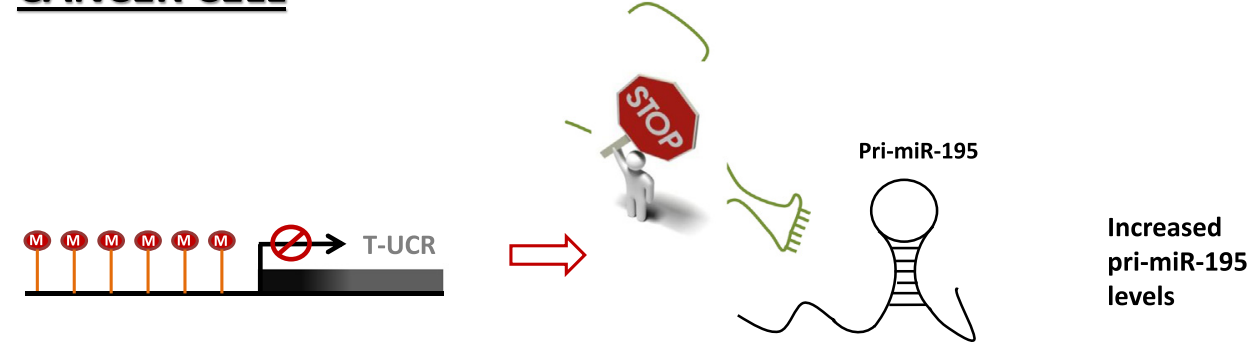

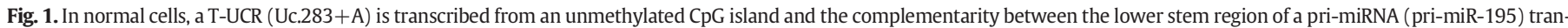

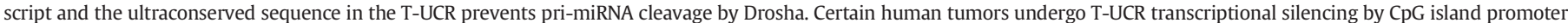
hypermethylation and the production of mature miR-195 cannot be regulated.

neuroblastoma. A thorough analysis of the expression of the 481 T-UCRs and 723 microRNAs in 34 neuroblastoma patients led them to conclude that the complementary regulatory mechanism between T-UCRs and microRNAs and the resulting T-UCR expression pattern predicted outcome in high-risk neuroblastomas [89]. In the case of pediatric neuroblastoma tumors it should be noted that T-UCRs, in addition to protein-coding genes located in CAGRs sites, may be involved in cancer susceptibility, since their expression is positively correlated with copynumber status [90].

\section{MicroRNA targets for T-UCRs}

The functional consequence of microRNA activity on T-UCRs (or on IncRNAs more generally) was not entirely surprising, given the high similarity of mRNA and lncRNA sequences. By contrast, the reciprocal regulation between the two classes of RNA is almost certain to yield more promising functions of lncRNAs, in addition to already known mechanisms.

Several lines of research have resulted in progress being made towards identifying functional interactions between IncRNAs as a new post-transcriptional mechanism in the control of microRNA function. Despite the importance of T-UCR expression in health and disease, the biological role and functional aspects of the vast majority of T-UCRs remain largely unknown. As described above, only one case of lncRNA derived from an ultraconserved region of the genome has so far been identified as a post-transcriptional modulator of microRNA function [22]. This new mode of regulation differs from the mode of action of other ceRNAs because the titration of microRNA from their respective targets does not necessarily reduce microRNA levels. In this particular case, the overexpression with the epigenetically disrupted Uc.283+A in HCT-116 colorectal cancer cells impairs the proper processing of miR-195 (Fig. 1). The regulation is based on the complementarity between the 11 nucleotides upstream of the Drosha cropping site and the 11 consecutive nucleotides within the ultraconserved sequence of the Uc.283+A. Despite not being sequence-dependent, the regulation requires complementarity between molecules, which implies that disturbances in the lower stem region of pri-miR-195 underpin the negative regulation. It is well known that the lower-strand region of
pri-microRNAs is crucial to DGCR8 recognition and the subsequent catalysis mediated by the endonuclease Drosha. As a result, and as validated in vivo and in vitro, the presence of the complementarity between Uc.283+A and pri-miR-195 reduces the production of mature and functional miR-195 molecules, which in turn leads to the derepression of a functional target in vivo. It would be interesting to settle this point, since many miR-195 targets are closely related to proliferation and cell cycle progression [91,92], and Uc.283 + A is highly expressed in neuroblastoma tumors [90].

Currently, much effort is being made to elucidate the wellorchestrated mechanisms that underlie the various regulatory networks controlling cell physiology in health and disease. This review summarizes some recent examples that describe the concept of IncRNA:microRNA interactions, which operate spatiotemporally during tumorigenic processes. Taken together, microRNAs and lncRNAs cooperate with each other to fine-tune gene expression at the transcriptional and post-transcriptional levels. It is clear that these noncoding RNAs species influence every aspect of cell physiology through a sophisticated and multi-layered mode of regulation, even though we currently lack information about the mechanistic aspects underlying the regulatory interactions. Recent advances have revealed fascinating new modes of interaction between non-coding RNA molecules that were once dismissed as being part of transcriptional noise. For instance, in the next few years, the analysis of RNA-RNA or RNA-protein interactions, such us those that include the Ago and RNA binding proteins, by CLASH [93], iCLIP [94], HITS-CLIP [94,95], as well as the integration of in vivo RNA structural analysis [96], seem likely to produce an expansion in our understanding of how the interaction between non-coding RNA molecules affects normophysiology and pathophysiology.

\section{References}

[1] P. Carninci, et al., The transcriptional landscape of the mammalian genome, Science 309 (5740) (2005) 1559-1563.

[2] H. Ling, M. Fabbri, G.A. Calin, MicroRNAs and other non-coding RNAs as targets for anticancer drug development, Nat. Rev. Drug Discov. 12 (11) (2013) 847-865.

[3] Y.K. Kim, V.N. Kim, Processing of intronic microRNAs, EMBO J. 26 (3) (2007) 775-783. 
[4] Y. Hayashita, et al., A polycistronic microRNA cluster, miR-17-92, is overexpressed in human lung cancers and enhances cell proliferation, Cancer Res. 65 (21) (2005) 9628-9632.

[5] Y. Lee, et al., The nuclear RNase III Drosha initiates microRNA processing, Nature 425 (6956) (2003) 415-419.

[6] A.M. Denli, et al., Processing of primary microRNAs by the Microprocessor complex, Nature 432 (7014) (2004) 231-235.

[7] G. Hutvagner, et al., A cellular function for the RNA-interference enzyme Dicer in the maturation of the let-7 small temporal RNA, Science 293 (5531) (2001) 834-838.

[8] D.S. Schwarz, et al., Asymmetry in the assembly of the RNAi enzyme complex, Cell 115 (2) (2003) 199-208

[9] J. Liu, et al., Argonaute2 is the catalytic engine of mammalian RNAi, Science 305 (5689) (2004) 1437-1441.

[10] H. Zhou, I. Rigoutsos, MiR-103a-3p targets the 5' UTR of GPRC5A in pancreatic cells, RNA 20 (9) (2014) 1431-1439.

[11] A. Eulalio, E. Huntzinger, E. Izaurralde, GW182 interaction with Argonaute is essential for microRNA-mediated translational repression and mRNA decay, Nat. Struct. Mol. Biol. 15 (4) (2008) 346-353.

[12] Y. Saito, et al., Specific activation of microRNA-127 with downregulation of the proto-oncogene BCL6 by chromatin-modifying drugs in human cancer cells, Cancer Cell 9 (6) (2006) 435-443.

[13] Y.S. Lee, A. Dutta, MicroRNAs in cancer, Annu. Rev, Pathol. 4 (2009) 199-227.

[14] K.A. O'Donnell, et al., c-Myc-regulated microRNAs modulate E2F1 expression, Nature 435 (7043) (2005) 839-843.

[15] L. He, et al., A microRNA component of the p53 tumour suppressor network, Nature 447 (7148) (2007) 1130-1134.

[16] A. Lujambio, et al., A microRNA DNA methylation signature for human cancer metastasis, Proc. Natl. Acad. Sci. U. S. A. 105 (36) (2008) 13556-13561.

[17] M.R. Suh, et al., Human embryonic stem cells express a unique set of microRNAs, Dev. Biol. 270 (2) (2004) 488-498.

[18] A. Shenoy, R.H. Blelloch, Regulation of microRNA function in somatic stem cell proliferation and differentiation, Nat. Rev. Mol. Cell Biol. 15 (9) (2014) 565-576.

[19] M. Ha, V.N. Kim, Regulation of microRNA biogenesis, Nat. Rev. Mol. Cell Biol. 15 (8) (2014) 509-524

[20] S. Guil, J.F. Caceres, The multifunctional RNA-binding protein hnRNP A1 is required for processing of miR-18a, Nat. Struct. Mol. Biol. 14 (7) (2007) 591-596.

[21] N.R. Choudhury, et al., Tissue-specific control of brain-enriched miR-7 biogenesis Genes Dev. 27 (1) (2013) 24-38.

[22] J. Liz, et al., Regulation of pri-microRNA processing by a long noncoding RNA transcribed from an ultraconserved region. Mol. Cell 55 (1) (2014) 138-147.

[23] J.M. Thomson, et al., Extensive post-transcriptional regulation of microRNAs and its implications for cancer, Genes Dev. 20 (16) (2006) 2202-2207.

[24] C. Kandoth, et al., Mutational landscape and significance across 12 major cancer types, Nature 502 (7471) (2013) 333-339.

[25] G. Ciriello, et al., Emerging landscape of oncogenic signatures across human cancers, Nat. Genet. 45 (10) (2013) 1127-1133.

[26] M.K. Iyer, et al., The landscape of long noncoding RNAs in the human transcriptome Nat. Genet. 47 (3) (2015) 199-208.

[27] D. Vucicevic, et al., Long ncRNA expression associates with tissue-specific enhancers, Cell Cycle 14 (2) (2015) 253-260.

[28] S. Diederichs, The four dimensions of noncoding RNA conservation, Trends Genet. 30 (4) (2014) 121-123.

[29] S. Feng, et al., Expression and functional role of reprogramming-related long noncoding RNA (lincRNA-ROR) in glioma, J. Mol. Neurosci. 56 (3) (2015) 623-630.

[30] L. Hu, et al., Up-regulation of long noncoding RNA MALAT1 contributes to proliferation and metastasis in esophageal squamous cell carcinoma, J. Exp. Clin. Cancer Res. 34 (1) (2015) 7.

[31] N. Brockdorff, Noncoding RNA and polycomb recruitment, RNA 19 (4) (2013) 429-442.

[32] M. Beltran, et al., A natural antisense transcript regulates Zeb2/Sip1 gene expression during Snail1-induced epithelial-mesenchymal transition, Genes Dev. 22 (6) (2008) 756-769.

[33] F. Guo, et al., Inhibition of metastasis-associated lung adenocarcinoma transcript 1 in CaSki human cervical cancer cells suppresses cell proliferation and invasion, Acta Biochim. Biophys. Sin. 42 (3) (2010) 224-229 (Shanghai)

[34] C. Gong, L.E. Maquat, IncRNAs transactivate STAU1-mediated mRNA decay by duplexing with 3' UTRs via Alu elements, Nature 470 (7333) (2011) 284-288.

[35] K. Panzitt, et al., Characterization of HULC, a novel gene with striking up-regulation in hepatocellular carcinoma, as noncoding RNA, Gastroenterology 132 (1) (2007) 330-342.

[36] J. Jendrzejewski, et al., The polymorphism rs944289 predisposes to papillary thyroid carcinoma through a large intergenic noncoding RNA gene of tumor suppressor type, Proc. Natl. Acad. Sci. U. S. A. 109 (22) (2012) 8646-8651.

[37] P. Johnsson, et al., A pseudogene long-noncoding-RNA network regulates PTEN transcription and translation in human cells, Nat. Struct. Mol. Biol. 20 (4) (2013) 440-446.

[38] X. Yang, et al., The emergence of long non-coding RNAs in the tumorigenesis of hepatocellular carcinoma, Cancer Lett. 360 (2) (2015) 119-124.

[39] F. Chen, et al., MALAT2-activated long noncoding RNA indicates a biomarker of poor prognosis in gastric cancer, Cancer Gene Ther. (2015) http://dx.doi.org/10.1038/cgt 2015.6.

[40] L. Han, et al., Low expression of long noncoding RNA PANDAR predicts a poor prognosis of non-small cell lung cancer and affects cell apoptosis by regulating Bcl-2, Cell Death Dis. 6 (2015) e1665.

[41] S. Emmrich, et al., LincRNAs MONC and MIR100HG act as oncogenes in acute megakaryoblastic leukemia, Mol. Cancer 13 (2014) 171
[42] T. Chiyomaru, et al., Genistein inhibits prostate cancer cell growth by targeting miR34a and oncogenic HOTAIR, PLoS One 8 (8) (2013) e70372.

[43] M.T. Qiu, et al., Long noncoding RNA: an emerging paradigm of cancer research, Tumour Biol. 34 (2) (2013) 613-620.

[44] P. Wang, et al., Long non-coding RNA CASC2 suppresses malignancy in human gliomas by miR-21, Cell. Signal. 27 (2) (2015) 275-282.

[45] A. Lujambio, et al., CpG island hypermethylation-associated silencing of non-coding RNAs transcribed from ultraconserved regions in human cancer, Oncogene 29 (48) (2010) 6390-6401.

[46] F.H. Tsang, et al., Long non-coding RNA HOTTIP is frequently up-regulated in hepatocellular carcinoma and is targeted by tumour suppressive miR-125b, Liver Int. 35 (5) (2014) 1597-1606.

[47] J.H. Yoon, et al., LincRNA-p21 suppresses target mRNA translation, Mol. Cell 47 (4) (2012) 648-655.

[48] E. Leucci, et al., microRNA-9 targets the long non-coding RNA MALAT1 for degradation in the nucleus, Sci. Rep. 3 (2013) 2535.

[49] X. Wang, et al., Silencing of long noncoding RNA MALAT1 by miR-101 and miR-217 inhibits proliferation, migration, and invasion of esophageal squamous cell carcinoma cells, J. Biol. Chem. 290 (7) (2015) 3925-3935.

[50] Q. Liu, et al., LncRNA loc285194 is a p53-regulated tumor suppressor, Nucleic Acids Res. 41 (9) (2013) 4976-4987.

[51] P. Baldinu, et al., CASC2a gene is down-regulated in endometrial cancer, Anticancer Res. 27 (1A) (2007) 235-243.

[52] Y. Han, et al., UCA1, a long non-coding RNA up-regulated in colorectal cancer influences cell proliferation, apoptosis and cell cycle distribution, Pathology 46 (5) (2014) 396-401.

[53] Z. Fang, et al., Increased expression of the long non-coding RNA UCA1 in tongue squamous cell carcinomas: a possible correlation with cancer metastasis, Oral Surg. Oral Med. Oral Pathol. Oral Radiol. 117 (1) (2014) 89-95.

[54] J. Huang, et al., Long non-coding RNA UCA1 promotes breast tumor growth by suppression of p27 (Kip1), Cell Death Dis. 5 (2014) e1008.

[55] Y. Fan, et al., Long non-coding RNA UCA1 increases chemoresistance of bladder cancer cells by regulating Wnt signaling, FEBS J. 281 (7) (2014) 1750-1758.

[56] T. Wang, et al., Hsa-miR-1 downregulates long non-coding RNA urothelial cancer associated 1 in bladder cancer, Tumour Biol. 35 (10) (2014) 10075-10084.

[57] T. Nagano, et al., The Air noncoding RNA epigenetically silences transcription by targeting G9a to chromatin, Science 322 (5908) (2008) 1717-1720.

[58] K.M. Schmitz, et al., Interaction of noncoding RNA with the rDNA promoter mediates recruitment of DNMT3b and silencing of rRNA genes, Genes Dev. 24 (20) (2010) 2264-2269.

[59] I. Martianov, et al., Repression of the human dihydrofolate reductase gene by a noncoding interfering transcript, Nature 445 (7128) (2007) 666-670.

[60] Y. Jeon, J.T. Lee, YY1 tethers Xist RNA to the inactive X nucleation center, Cell 146 (1) (2011) 119-133.

[61] K.C. Wang, et al., A long noncoding RNA maintains active chromatin to coordinate homeotic gene expression, Nature 472 (7341) (2011) 120-124.

[62] L. Quagliata, et al., Long noncoding RNA HOTTIP/HOXA13 expression is associated with disease progression and predicts outcome in hepatocellular carcinoma patients, Hepatology 59 (3) (2014) 911-923.

[63] T. Hung, et al., Extensive and coordinated transcription of noncoding RNAs within cell-cycle promoters, Nat. Genet. 43 (7) (2011) 621-629.

[64] P.K. Puvvula, et al., Long noncoding RNA PANDA and scaffold-attachment-factor SAFA control senescence entry and exit, Nat. Commun. 5 (2014) 5323.

[65] W. Yu, et al., Epigenetic silencing of tumour suppressor gene p15 by its antisense RNA, Nature 451 (7175) (2008) 202-206.

[66] E. Pasmant, et al., Characterization of a germ-line deletion, including the entire INK4/ARF locus, in a melanoma-neural system tumor family: identification of ANRIL, an antisense noncoding RNA whose expression coclusters with ARF, Cancer Res. 67 (8) (2007) 3963-3969.

[67] F.Q. Nie, et al., Long noncoding RNA ANRIL promotes non-small cell lung cancer cell proliferation and inhibits apoptosis by silencing KLF2 and P21 expression, Mol. Cancer Ther. 14 (1) (2015) 268-277.

[68] Y. Ding, et al., In vivo genome-wide profiling of RNA secondary structure reveals novel regulatory features, Nature 505 (7485) (2014) 696-700.

[69] N. Liu, et al., N(6)-methyladenosine-dependent RNA structural switches regulate RNA-protein interactions, Nature 518 (7540) (2015) 560-564.

[70] L. Yang, et al., IncRNA-dependent mechanisms of androgen-receptor-regulated gene activation programs, Nature 500 (7464) (2013) 598-602.

[71] J.M. Franco-Zorrilla, et al., Target mimicry provides a new mechanism for regulation of microRNA activity, Nat. Genet. 39 (8) (2007) 1033-1037.

[72] H. Seitz, Redefining microRNA targets, Curr. Biol. 19 (10) (2009) 870-873.

[73] L. Poliseno, et al., A coding-independent function of gene and pseudogene mRNAs regulates tumour biology, Nature 465 (7301) (2010) 1033-1038.

[74] A. Gabory, H. Jammes, L. Dandolo, The H19 locus: role of an imprinted non-coding RNA in growth and development, Bioessays 32 (6) (2010) 473-480.

[75] C. Ma, et al., H19 promotes pancreatic cancer metastasis by derepressing let-7's suppression on its target HMGA2-mediated EMT, Tumour Biol. 35 (9) (2014) 9163-9169.

[76] A.N. Kallen, et al., The imprinted H19 IncRNA antagonizes let-7 microRNAs, Mol. Cell 52 (1) (2013) 101-112.

[77] M. Fan, et al., A long non-coding RNA, PTCSC3, as a tumor suppressor and a target of microRNAs in thyroid cancer cells, Exp. Ther. Med. 5 (4) (2013) $1143-1146$

[78] Y. Gao, et al., LncRNA-HOST2 regulates cell biological behaviors in epithelial ovarian cancer through a mechanism involving microRNA let-7b, Hum. Mol. Genet. 24 (3) (2015) 841-852. 
[79] Y. Wang, et al., Endogenous microRNA sponge lincRNA-RoR regulates Oct4, Nanog, and Sox2 in human embryonic stem cell self-renewal, Dev. Cell 25 (1) (2013) 69-80.

[80] X. Zhou, et al., Linc-RNA-RoR acts as a "sponge" against mediation of the differentiation of endometrial cancer stem cells by microRNA-145, Gynecol. Oncol. 133 (2) (2014) 333-339.

[81] J. Wang, et al., CREB up-regulates long non-coding RNA, HULC expression through interaction with microRNA-372 in liver cancer, Nucleic Acids Res. 38 (16) (2010) 5366-5383.

[82] J.L. Franklin, et al., Malignant transformation of colonic epithelial cells by a colonderived long noncoding RNA, Biochem. Biophys. Res. Commun. 440 (1) (2013) 99-104.

[83] G. Bejerano, et al., Ultraconserved elements in the human genome, Science 304 (5675) (2004) 1321-1325.

[84] G.A. Calin, et al., Ultraconserved regions encoding ncRNAs are altered in human leukemias and carcinomas, Cancer Cell 12 (3) (2007) 215-229.

[85] C. Braconi, et al., Expression and functional role of a transcribed noncoding RNA with an ultraconserved element in hepatocellular carcinoma, Proc. Natl. Acad. Sci. U. S. A. 108 (2) (2011) 786-791.

[86] R.S. Hudson, et al., Transcription signatures encoded by ultraconserved genomic regions in human prostate cancer, Mol. Cancer 12 (2013) 13.

[87] J. Sana, et al., Expression levels of transcribed ultraconserved regions uc.73 and uc.388 are altered in colorectal cancer, Oncology 82 (2) (2012) 114-118.
[88] D. Ng, et al., Identification of a novel chromosome region, 13q21.33-q22.2, for susceptibility genes in familial chronic lymphocytic leukemia, Blood 109 (3) (2007) 916-925.

[89] P. Scaruffi, et al., Transcribed-ultra conserved region expression is associated with outcome in high-risk neuroblastoma, BMC Cancer 9 (2009) 441.

[90] P. Mestdagh, et al., An integrative genomics screen uncovers ncRNA T-UCR functions in neuroblastoma tumours, Oncogene 29 (24) (2010) 3583-3592.

[91] L. Liu, et al., microRNA-195 promotes apoptosis and suppresses tumorigenicity of human colorectal cancer cells, Biochem. Biophys. Res. Commun. 400 (2) (2010) 236-240.

[92] T. Xu, et al., MicroRNA-195 suppresses tumorigenicity and regulates G1/S transition of human hepatocellular carcinoma cells, Hepatology 50 (1) (2009) 113-121.

[93] A. Helwak, D. Tollervey, Mapping the microRNA interactome by cross-linking ligation and sequencing of hybrids (CLASH), Nat. Protoc. 9 (3) (2014) 711-728.

[94] C. Yao, L. Weng, Y. Shi, Global protein-RNA interaction mapping at single nucleotide resolution by iCLIP-seq, Methods Mol. Biol. 1126 (2014) 399-410.

[95] J.H. Yang, L.H. Qu, Discovery of microRNA regulatory networks by integrating multidimensional high-throughput data, Adv. Exp. Med. Biol. 774 (2013) 251-266.

[96] C.K. Kwok, et al., Determination of in vivo RNA structure in low-abundance transcripts, Nat. Commun. 4 (2013) 2971.

[97] P. Hou, et al., LincRNA-ROR induces epithelial-to-mesenchymal transition and contributes to breast cancer tumorigenesis and metastasis, Cell Death Dis. 5 (2014) e1287. 